\title{
Fast Two-Qubit Gates in Semiconductor Quantum Dots using a Photonic Microcavity
}

\author{
Dmitry Solenov, ${ }^{1}$ Sophia E. Economou, ${ }^{1,}$ 用 and T. L. Reinecke ${ }^{1}$ \\ ${ }^{1}$ Naval Research Laboratory, Washington, District of Columbia 20375, USA
}

\begin{abstract}
Implementations for quantum computing require fast single- and multi-qubit quantum gate operations. In the case of optically controlled quantum dot qubits theoretical designs for long-range two- or multi-qubit operations satisfying all the requirements in quantum computing are not yet available. We have developed a design for a fast, long-range two-qubit gate mediated by a photonic microcavity mode using excited states of the quantum dot-cavity system that addresses these needs. This design does not require identical qubits, it is compatible with available optically induced single qubit operations, and it advances opportunities for scalable architectures. We show that the gate fidelity can exceed $90 \%$ in experimentally accessible systems.
\end{abstract}

\section{INTRODUCTION}

Quantum information processing involves the manipulation of entanglement carried out by unitary gate operations between different quantum bits (qubits). Realistic quantum computing architectures require entangling gates between distant qubits. Optical photons provide a natural vehicle to implement such interactions in many physical systems $!^{1}$ As a result, architectures based on optically active qubits that can couple to photonic modes in optical cavities and waveguides, such as quantum dots, NV centers, and trapped ions are attractive for large scale quantum computing. ${ }^{25}$ Quantum dots (QDs) in particular hold promise as qubits for such architectures, in part owing to their large dipole moments, which allow them to couple efficiently to the optical cavity modes and to photonic flying qubits for extended architectures. Qubits encoded by the spin of an electron in a QD have long coherence times which are five to six orders of magnitude longer than the typical picosecond scale of optical control. Successful initialization and readout, as well as fast optical single spin rotations, have been demonstrated in these systems $\frac{617}{}$ In addition, important advances have recently been achieved in work on coupled cavity-QD systems, including demonstrations of

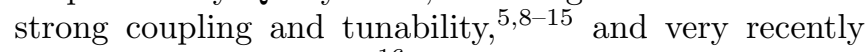
full single-qubit control! 16

A critical step needed to advance the field is the design of a two-qubit controlled gate operation mediated by an optical cavity mode. A viable two-qubit quantum gate requires that several criteria are met: (i) a longrange switchable physical interaction between qubits is available; (ii) the gate performs a unitary operation on one qubit depending on the state of the other qubit to provide a controlled operation; (iii) the operations are sufficiently fast compared to decoherence rates; (iv) the gate is compatible with single-qubit rotations (to form a universal set of gates); (v) the gate design is consistent with a multi-qubit system for scalability.

So far, only local control of entanglement in closely spaced quantum dots (QD 'molecules') has been demonstrated experimentally 17 For an experimental demonstration of cavity-mediated entangling gates, a theoretical design is needed that satisfies the above criteria, (i)- (v), while being experimentally simple and compatible with current technology. Existing proposals for cavitymediated gates have not met these requirements; they are either incompatible with single-qubit gates, $\frac{18}{18}$ limited to nearest-neighbor qubits, $\frac{19}{19}$ and/or require adiabaticity, either through adiabatic evolution $\frac{19}{19}$ or through adiabatic elimination of the auxiliary state ${ }^{[2]}$ As a result, they are much slower than what is needed from a quantum information processing perspective. Moreover, a careful assessment of the performance of such gates as a function of system parameters has not been given in the literature, despite the key role it would play in experimental demonstrations.

In this paper we give a novel design for an entangling control-z (CZ) two-qubit gate ${ }^{21}$ that satisfies all the above criteria. Our design does not require the QD energies to be equal or dynamically tunable. As a result, our approach is compatible with single qubit operations and has a potential for many-qubit scalable architectures. We obtain fidelities in excess of $90 \%$ for realistic parameters. In the following we explain the concept of this all-optical gate, formulate the model, calculate the QD-cavity system spectrum, and analyze our design of the two-qubit gate protocol. The fidelity of the gate operation as a function of the system parameters is also calculated and provides a guide for experiment.

\section{TWO-QUBIT GATES}

The control-z gate is a maximally entangling two-qubit gate, and it is given by $U_{C Z}=\operatorname{diag}(1,1,1,-1)$. It is equivalent to the more familiar control-NOT (CNOT) operation up to single-qubit gates. Specifically, $U_{C N O T}=$ $(1 \otimes \mathrm{H}) U_{C Z}(1 \otimes \mathrm{H})$, where $H=\left(\begin{array}{cc}1 & 1 \\ 1 & -1\end{array}\right) / \sqrt{2}$ is the Hadamard gate. To see the entangling capability of $\mathrm{CZ}$ we can look at its action on a product state of two qubits. In particular, when each qubit is in an equal superposition of the basis states, we have

$U_{C Z}(|1\rangle+|0\rangle) \otimes(|1\rangle+|0\rangle)=|11\rangle+|10\rangle+|01\rangle-|00\rangle$,

which is a maximally entangled two-qubit state, also known as a two-qubit 'cluster state'. Such a state is equivalent to a Bell state up to single-qubit rotations. 
To implement the $\mathrm{CZ}$ gate, we need to accumulate a phase factor of -1 selectively to only one of the two-qubit basis states, taken to be $|00\rangle$ above. Meanwhile, to be able to perform single-qubit gates, the transition involving state $|00\rangle$ and an auxiliary state should be performed in parallel with that involving $|01\rangle$ (or $|10\rangle$ for rotations of the second qubit) and its corresponding auxiliary state. To avoid dynamically tuning energies-a process that is costly in time and can compete with coherence timeswe will use different classes of auxiliary states for singlequbit and two-qubit operations. In particular, we will use a near-resonance between the two-photon state of the cavity and the state where both QDs are excited.

\section{QUANTUM DOTS IN A CAVITY}

We focus on a system of two (singly) charged selfassembled InAs QDs in a photonic crystal microcavity. This structure can support in- and out-of-plane polarizations. ${ }^{[22}$ Due to strain the optical dipole transition matrix elements in the InAs dots are anisotropic, resulting in efficient absorption of light with electric field polarization perpendicular to the QD growth axis. As a result, only the mode with electric field polarized in the plane of the crystal can be coupled to transitions in QDs. We take an external magnetic field to be applied in-plane (Voigt configuration), perpendicular to the QD growth direction. This will enable full single qubit control as explained in Ref. 20.

The system can be represented by two separate fourstate QDs interacting with a single photon mode, as shown in Fig. 1(a). The two lowest energy states of each four-state system are the spin states of the electron in each dot, which represent the qubit, $|\uparrow\rangle=c_{n, \uparrow}^{\dagger}|\rangle_{0}$ and $|\downarrow\rangle=c_{n, \downarrow}^{\dagger}|\rangle_{0}$, where $n=1,2$ refers to the two dots and $c_{\uparrow(\downarrow)}^{\dagger}$ creates an electron of spin $\uparrow(\downarrow)$ relative to the uncharged QD state |\rangle$_{0}$. The two excited states in each dot are electron-exciton bound states, called trions (or charged excitons). They are complexes having total angular momentum $3 / 2$. The two $\pm 3 / 2$ states ('heavy holes') are energetically lower than the $\pm 1 / 2$ ('light hole') states and thus form a pseudo spin $|\Uparrow\rangle=t_{n, \uparrow}^{\dagger}|\rangle_{0}=c_{n, \uparrow}^{\dagger} c_{n, \downarrow}^{\dagger} h_{n, \Uparrow}^{\dagger}|\rangle_{0}$ and similarly for $|\Downarrow\rangle$, where $h^{\dagger}$ is the creation operator for a heavy hole. The trion states carry the pseudospin of the hole because the two electrons are in a spin singlet. We choose the spin quantization axis along the external magnetic field.

The cavity couples to the trion transitions and preserves the (pseudo) spin orientation, $|\uparrow\rangle \leftrightarrow|\uparrow\rangle$ and $|\downarrow\rangle \leftrightarrow$ $|\downarrow\rangle$. In the rotating-wave approximation the cavity-dot interaction is

$$
H_{Q D-C}=g \sum_{n=1,2}\left(t_{n, \uparrow}^{\dagger} c_{n, \uparrow} a+t_{n, \downarrow}^{\dagger} c_{n, \downarrow} a+h . c .\right)
$$

where $a$ annihilates a photon in the cavity and $g$ is the coupling between the trion transition and the cavity. We

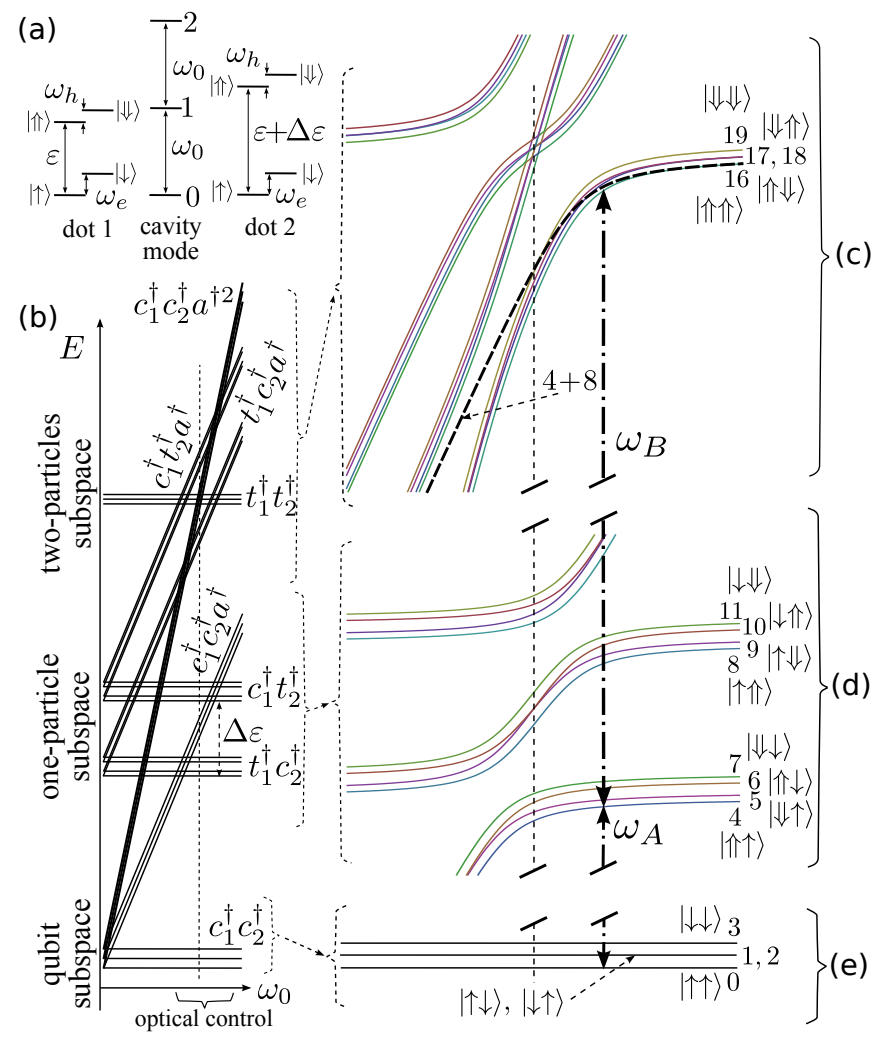

FIG. 1: The cavity-dot system. (a) Energies and relevant states of two QDs and cavity. (b-e) Interacting cavity-dot spectrum as a function of the cavity mode frequency, $\omega_{0}$. (b) Structure of crossings and corresponding states. Panels (c) and (d) show the anti-crossing splittings in the two- and single-excitation subspaces respectively. Panel (e) shows the energy structure of the qubit subspace, which is unaffected by the coupling to the cavity mode. The numbers in (c-e) give the states of the diagonalized Hamiltonian, and the $\uparrow, \downarrow$ show the predominant spin character of each state far (to the right) of the avoided crossings. Vertical dashed lines indicate $\left(\varepsilon_{1}+\varepsilon_{2}\right) / 2$.

choose these coupling constants to be the same for the two QDs to simplify the presentation. This assumption is not important to the proposed procedure and can be relaxed when necessary.

The spectrum of the cavity-QD system is shown in Fig. 1 as a function of the cavity frequency $\omega_{0}$. This representation does not suggest the need to tune $\omega_{0}$ dynamically, but it helps to identify the region of optimal $\omega_{0}$ values. The spectrum is obtained by diagonalizing $H_{0}=$ $H_{Q D}+H_{C}+H_{Q D-C}$, where $H_{Q D}=\sum_{n, \xi} \omega_{e} \theta(\xi) c_{n, \xi}^{\dagger} c_{n, \xi}+$ $\sum_{n, \xi} t_{n, \xi}^{\dagger}\left[\varepsilon_{n}+\omega_{h} \theta(\xi)\right] t_{n, \xi}, H_{C}=\omega_{0} a^{\dagger} a, \xi=\uparrow, \downarrow, \theta(\uparrow)=0$, and $\theta(\downarrow)=1$.

The Hamiltonian $H_{0}$ conserves the total number of excitations. As a result the Hilbert space of the system separates into subspaces with different numbers of excitations. Each subspace contains several states, corresponding to different spin projections. The lowest set of four states (three energy levels) defines the two-qubit 
subspace, $|\uparrow \uparrow\rangle,|\uparrow \downarrow\rangle,|\downarrow \uparrow\rangle$, and $|\downarrow \downarrow\rangle$, and has zero cavity photons; we call this the 'zero excitation' subspace. The other relevant subspaces are the 'one-excitation' subspace, that has states with either one cavity photon or one trion, and the 'two-excitation' subspace, that has states with two trions (one per dot), states with one trion and one cavity photon, and states with two cavity photons; see Appendix A. States in the 'one-excitation' part of the spectrum are approximately local to each quantum dot and interact with each other only very weakly, $\sim(g / \Delta \varepsilon)^{2}$. They are the states that can be used for single-qubit control. ${ }^{23}$ The two-excitation subspace involves hybridized states of the two QDs and are ideal for a two-qubit gate. These states however are not directly accessible from the qubit subspace with a single pulse, so we make use of a series of control pulses.

The laser pulses have momentum perpendicular to the photonic crystal plane to avoid Bragg shielding due to the photonic crystal. For definiteness we choose pulses with the same linear polarization as the cavity mode, $\stackrel{24}{,}$

$$
\mathcal{V}(t)=\sum_{p ; n>m} \Omega_{p}\left(t-t_{p}\right) 2 \cos \omega_{p} t\left(M_{n m}|n\rangle\langle m|+h . c .\right) .
$$

The total Hamiltonian becomes $\mathcal{H}(t)=\mathcal{H}_{0}+\mathcal{V}(t)$, where $\mathcal{H}_{0}=U^{\dagger} H_{0} U=\sum_{n} E_{n}|n\rangle\langle n|$ and $M_{n, m}=$ $\sum_{j=1,2, \xi}\left\langle n\left|U^{\dagger}\left(t_{j, \xi}^{\dagger} c_{j, \xi}+c_{j, \xi}^{\dagger} t_{j, \xi}\right) U\right| m\right\rangle$. The subscript $p$ enumerates the pulses used to perform the gate where each has frequency $\omega_{p}$ and is centered at time $t_{p}$.

\section{IMPLEMENTATION OF CZ GATE}

The CZ gate has a simple diagonal form, which allows for a relatively straightforward design based on phases induced by resonant cyclic excitation of an auxiliary excited state. The idea is to use the property of quantum two-level systems, in which a cyclic evolution from the ground state to the excited state and back to the ground state induces a minus sign to the latter. In the presence of additional, uncoupled states the minus sign is relative and thus constitutes a nontrivial quantum evolution. The pulse performing such an evolution is known as a ' $2 \pi$ ' pulse. Optical $2 \pi$ pulses were proposed theoretically for single-qubit rotations in quantum dots $\frac{23}{23}$ and two-qubit gates in quantum dot molecules 25 and later used in their experimental demonstrations. ${ }^{7 / 17}$

In our approach, the phase accumulation will be on state $|\uparrow \downarrow\rangle$, while keeping the phases of other basis states unchanged. This can be done by the following pulse sequence: (i) a population inversion $\pi$ pulse, pulse $\mathrm{A}$, tuned to transition $\omega_{1}=\omega_{A}=E_{4}-E_{0}$ between qubit state $|\uparrow \uparrow\rangle$ and the excited state with similar spin configuration, see Fig 11(d)-(e). The pulse is also in resonance with $E_{6}-E_{2}$ transition, and thus it creates a trion in the first QD only: both $|\uparrow \uparrow\rangle$ and $|\uparrow \downarrow\rangle$ are transformed in the same way and accumulate a phase factor of $-i$ each. (ii) A $2 \pi$, or phase, pulse (pulse B) with frequency $\omega_{2}=\omega_{B}=E_{16}-E_{4}$, see Fig 1 (c)-(d). This induces a transition between the 'one-excitation' states previously created and one of the 'two-excitation' states. Note that if $g=0$ or we are far detuned, $\omega \gg \Delta \varepsilon$, the transition $E_{10}-E_{2}$ would also occur. This would correspond to a single qubit operation on the second qubit, i.e., $|\Uparrow \uparrow\rangle$ and $|\downarrow \uparrow\rangle$ would both acquire a phase factor of -1 . A nonzero $g$ induces formation of two-excitation states that have different energies, c.f. the energy of state 16 and the sum of energies of states 4 and 8 . As a result, the state $\Uparrow \uparrow$, or state 4 , acquires the factor of -1 after the pulse, while state $|\downarrow \uparrow\rangle$ does not. (iii) Finally, we apply the population inversion pulse A again, $\omega_{3}=\omega_{A}$, to restore the system to the qubit subspace. This gives additional factors of $-i$ to both $|\uparrow \uparrow\rangle$ and $|\uparrow \downarrow\rangle$, as mentioned above. The two phase factors of $(-i)$ induce a minus sign to states $|\uparrow \downarrow\rangle$ and $|\uparrow \uparrow\rangle$, while the $2 \pi$ pulse cancels that sign in state $|\uparrow \uparrow\rangle$. The phase between the control pulses $\mathrm{A}$ and $\mathrm{B}$ does not enter the result and therefore pulses with unequal frequencies do not have to be phase locked, which is a significant experimental convenience.

A physical explanation of this approach is the following: because each QD is off-resonant from the cavity, when only one of the QDs is excited and no other excitations are present in the system the excited QD can be roughly thought of as isolated, i.e., decoupled from the cavity and from the other QD. Thus, single excitations can implement single-qubit operations without disturbing the rest of the system. On the other hand, when both QDs are excited they are closer to the resonance with the cavity state. As a result, there is a large mixing between cavity states and the states of both QDs. Thus, using the two-excitation regime is a natural venue for performing two-qubit conditional operations while maintaining the ability to manipulate each QD spin separately.

\section{FIDELITY}

Now we consider the gate fidelity, which is a measure of how close our operation is to the target gate. There are two types of fidelity losses, those caused by unintended coherent dynamics due to coupling of the lasers to off-resonance transitions and those originating from random processes such as trion recombination. First we focus on the former mechanism. The unintended transitions can cause $U_{g}$ to deviate from the ideal $U_{C Z}$ and effectively cause loss of coherence in the qubit-subspace, even though the entire operation involving excited states is unitary and coherent.

To analyze this type of decoherence we compute the average fidelity, $F$, of the gate operation, as explained in detail in Appendix B including transitions 0-4, 4-6, 2-5, $3-7,0-8,2-10,1-9,3-11$ for pulse $A$ and transitions $4-16$, $6-18,0-8,2-10,1-9,3-11$ for pulse B. Other transitions are negligible either due to vanishing matrix elements or to large detuning. We chose different pulse widths for pulse $\mathrm{A}$ and pulse $\mathrm{B} \sigma_{A}=2 \sigma_{B}=2 \sigma$. In Fig. 2(a) 

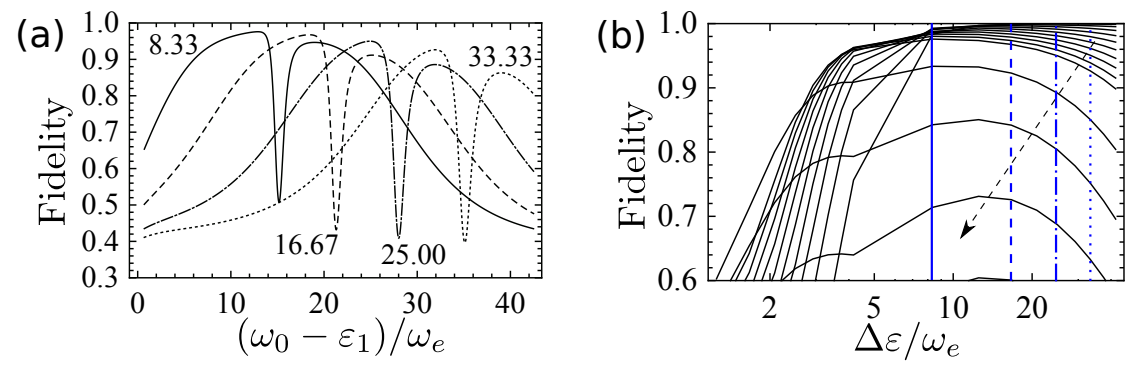

FIG. 2: Fidelity of CZ gate with imperfections resulting from coupling of the pulses to neighboring off-resonance optical transitions (a) as a function of $\omega_{0}$ for $\sigma / \omega_{e}=0.1$ for $\Delta \varepsilon / \omega_{e}=8.33,16.67,25.00,33.33$, as indicated, and (b) as a function of the spectral separation $\Delta \varepsilon$ between the QDs for different values of the pulse bandwidth, $\sigma / \omega_{e}=0.01,0.02, \ldots, 0.1,0.15, \ldots, 0.3$ as indicated by the dashed arrow. Each point is computed for the optimal value of $\omega_{0}$ from Fig. 2(a). The vertical lines mark the values of $\omega_{2}$ from panel (a). In both panels (a) and (b) we used $g / \omega_{e}=3.33$ and $\omega_{h}=\omega_{e} / 3$.

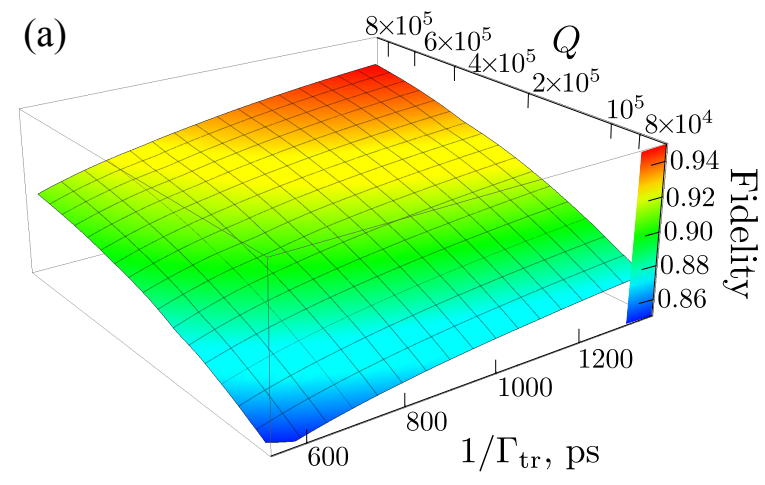

(b)

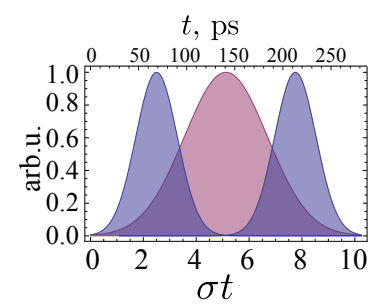

FIG. 3: (a) Fidelity of the two-qubit CZ gate in presence of decoherence due to trion recombination and cavity decay. The fidelity is plotted as the function of the trion decay time and the cavity mode quality factor. (b) The temporal profiles of the pulse sequence for $\sigma / \omega_{e}=0.2, \omega_{h}=\omega_{e} / 3, \Delta \varepsilon / \omega_{e}=8.33, g / \omega_{e}=3.33$, and $\omega_{e}=0.12 \mathrm{meV}$.

the fidelity is plotted as a function of the difference between the cavity mode frequency $\omega_{0}$ and the transition frequency of QD1 $\varepsilon_{1}$ for varying values of the frequency of QD2 $\varepsilon_{2}$. The qualitative features of the plots can be understood as follows: when the cavity mode frequency is much smaller or much larger than the QD frequencies, QD-cavity hybridization is negligible, and we are in a regime of two independent qubits. This causes attenuation of fidelity towards both sides of the plot. The dip in the middle occurs because, as the cavity is tuned, the target transition of pulse B (transition 4-16) becomes degenerate with transition $3-11$, and therefore state $|\downarrow \downarrow\rangle$ is also affected by pulse B, resulting in strong unintended dynamics. Note that at its high values the fidelity does not vary strongly with $\varepsilon_{1}$ and $\varepsilon_{2}$. As a result, gates between several different pairs of quantum dot spin qubits can be performed with high fidelity using only one cavity mode to mediate the interactions, which is an intriguing opportunity for scalable architectures.

Fig. 2(b) shows the fidelity as a function of the spectral separation $\Delta \varepsilon$ between the trions in QD1 and in QD2 for different pulse bandwidths $\sigma$. When $\Delta \varepsilon$ is small (comparable to $\omega_{e}$ ) the fidelity drops appreciably. This drop is the result of coupling in the 'one-excitation' subspace, i.e., the assumption that an excited QD is isolated from the rest of the system is no longer valid. Thus it also identifies the regime where fast optical single-qubit control is not possible. In the region of higher fidelities, where $\Delta \varepsilon / \omega_{e} \gtrsim 10$, the fidelity approaches its maximal value for longer pulses and starts decreasing more rapidly for $\sigma / \omega_{e} \gtrsim 0.2$ due to involvement of a larger number of unintended transitions.

Next, we consider the effects of decoherence due to losses during the gate. The main contributions come from trion recombination and cavity photon leakage. The typical linewidth of the trion state, $\Gamma_{\mathrm{tr}}$, in InAs QDs is $\sim 1 \mu \mathrm{eV} !^{26}$ The loss rate associated with the cavity is $\Gamma_{c}=\omega_{0} / Q$. State-of-the-art microcavities ${ }^{15}$ can have $Q$ 's up to $\sim 10^{5}$, which gives $\Gamma_{c} \sim 10 \mu \mathrm{eV}$. We calculate the fidelity using the standard master equation formalism 2127 and include states from 0 to 19 , see Appendix B The fidelity as a function of $Q$ and $1 / \Gamma_{\mathrm{tr}}$ in shown in Fig. 3 . It is maximized when the pulses overlap to reduce the time the excited states are occupied. We choose $\omega_{0}$ from the maximal fidelities, as in Fig. 22(a) for each point of Fig. 3. We see that fidelities in excess of $90 \%$ are possible for realistic values of the parameters. 


\section{CONCLUSIONS}

In summary, we have developed a design for a cavitymediated entangling gate between two spin qubits that satisfies the criteria for a realistic two-qubit operation. Our control-z gate is compatible with available single qubit operations and with natural inhomogeneities in optical resonances. It can thus accommodate several qubits that couple pairwise with appropriate control laser frequencies, opening a path to scalable architectures. It may also be useful for hybrid quantum computing approaches with various physical systems ${ }^{28}$ We have shown that the gate fidelity is at least $90 \%$ for current experimental parameters. Higher fidelities can be achieved in various ways such as using pulse shaping techniques $29 \mid 30$ and engineering higher finesse cavities.

\section{ACKNOWLEDGEMENTS}

This work was supported in part by NSA/LPS and in part by ONR.

\section{Appendix A: The spectrum}

In the rotating wave approximation the Hamiltonian [Eq. (1) from the main text] conserves the total number of excitations. Therefore it can be diagonalized independently in each excitation-number subspace. The lowest energy set of four states (three energy levels) corresponds to a subset with zero excitations. It represents the twoqubit subspace with zero cavity photons,

$$
0 \rightarrow|\uparrow \uparrow\rangle|0\rangle, 1 \rightarrow|\uparrow \downarrow\rangle|0\rangle, 2 \rightarrow|\downarrow \uparrow\rangle|0\rangle, 3 \rightarrow|\downarrow \downarrow\rangle|0\rangle,
$$

where $|0\rangle$ is the vacuum state of the cavity. The corresponding energies are controlled by the magnetic field via Zeeman splitting. For typical values of magnetic field of $\sim 1 \mathrm{~T}$ used in the initialization and readout and singlequbit experiments the splitting between $E_{0}, E_{3}$ and $E_{1,2}$ is $\sim 0.1 \mathrm{meV}$. The micro-cavity optical mode is coupled to the excitonic transitions in each quantum dot with the transition energies $\sim \mathrm{eV}$. As a result, the qubit subspace is not affected by the cavity.

The one-excitation subspace occurs at the optical frequency, $\sim \mathrm{eV}$ from the qubit subspace energies:

$$
\begin{aligned}
\operatorname{dot} 1: & |\uparrow \uparrow\rangle|0\rangle,|\downarrow \uparrow\rangle|0\rangle,|\Uparrow \downarrow\rangle|0\rangle,|\Downarrow \downarrow\rangle|0\rangle, \\
\operatorname{dot} 2: & |\uparrow \uparrow\rangle|0\rangle,|\uparrow \downarrow\rangle|0\rangle,|\downarrow \uparrow\rangle|0\rangle,|\downarrow \downarrow\rangle|0\rangle, \\
\text { cavity : } & |\uparrow \uparrow\rangle|1\rangle,|\uparrow \downarrow\rangle|1\rangle,|\downarrow \uparrow\rangle|1\rangle,|\downarrow \downarrow\rangle|1\rangle,
\end{aligned}
$$

where $|1\rangle$ denotes the state with a single photon in the cavity. The energy gap $\Delta \varepsilon$ between states A2 and A3 is due to the fact that the two dots are not identical in size, shape, and strain environment, which affects the excitonic transitions. The typical variation in trion transition energies is $\sim 1-20 \mathrm{meV}$. The energy of the cavity mode, $\omega_{0}$, is fixed during the gate operation but can be set to an optimal value during sample growth. The interaction with a cavity photon shifts the energies and mixes trion and photon states. The energies of the resulting states can be found analytically: note that states A2 A4 are always coupled in triplets. For example, the state $|\uparrow \uparrow\rangle|1\rangle$ interacts only with $|\uparrow \uparrow\rangle|0\rangle$ and $|\uparrow \uparrow\rangle|0\rangle$. For each triplet we have

$$
\left(E-\varepsilon_{1, \xi}\right)\left(E-\varepsilon_{2, \xi}\right)\left(E-\omega_{0}\right)=g^{2}\left(E-\varepsilon_{1, \xi}\right)+g^{2}\left(E-\varepsilon_{2, \xi}\right),(
$$

where $\xi=\uparrow$ or $\downarrow, \varepsilon_{n, \uparrow}=\varepsilon_{n}$ and $\varepsilon_{n, \downarrow}=\varepsilon_{n}+\omega_{h}-\omega_{e}$. Each triplet forms two anti-crossings when $\omega_{0}$ is swapped across the trion energies [see Fig. 1(b) and Fig. 1(d) of the main text]. When $g \sim \Delta \varepsilon$ or $g \gg \Delta \varepsilon$, the two excited quantum dot states can mix and form spin-entangled states. For experimentally accessible systems of quantum dots in a micro-cavity the coupling strength $g$ is substantially smaller than the variation in trion energies $\Delta \varepsilon$ and the mixing is negligible. In the limit $g \ll \Delta \varepsilon$ the interaction between one-excitation states from different QDs can be estimated by analyzing the difference $\delta \omega_{\uparrow}$ in transition energies between $\omega_{\uparrow}:|\uparrow \uparrow\rangle \rightarrow|\uparrow \uparrow\rangle$ and $\omega_{\uparrow}^{\prime}:|\uparrow \downarrow\rangle \rightarrow|\uparrow \downarrow\rangle$. From Eq. A5 we find $\omega_{\uparrow}=\varepsilon+g^{2} / f\left(\omega_{A}, \Delta \varepsilon\right)$ and $\omega_{\uparrow}^{\prime}=\varepsilon+g^{2} / f\left(\omega_{A}^{\prime}, \Delta \varepsilon+\omega_{e}-\omega_{h}\right)$, where $f(y, x)=$ $x-\omega_{0}-g^{2} /(x-\varepsilon+y)$. Since $\omega_{e} \sim \omega_{h} \sim g \ll \Delta \varepsilon$ it is easy to show that $\delta \omega_{\uparrow} \lesssim-g^{2} \omega_{e} / \Delta \varepsilon^{2}$. This should be compared to the typical inverse lifetime of the trion state, $\sim 1 \mu \mathrm{eV}$ (in energy units) or $\sim \omega_{e} / 100$. As a result for $\omega_{e} / \Delta \varepsilon \sim 10, \omega_{\uparrow}$ and $\omega_{\uparrow}^{\prime}$ are practically indistinguishable. This result is confirmed numerically by computing the spectrum (and the states) for different values of $\Delta \varepsilon$. It also holds for other transitions between the qubit and the one-excitation subspace states. Therefore we conclude that the one excitation subspace cannot be used for a two-qubit operations. It can, however, be used to perform fast single qubit operations as described in Ref. 23 by using the localized trion state.

In order to find useful non-local states that can mediate a two-qubit gate we investigate the two-excitation subspace. In this subspace the states are coupled in groups of four, e.g. $|\Uparrow \Uparrow, 0\rangle,|\Uparrow \uparrow, 1\rangle,|\uparrow \uparrow, 1\rangle,|\uparrow \uparrow, 2\rangle$ :

$$
\begin{gathered}
\left(\varepsilon_{2, \xi}+\varepsilon_{1, \xi}-E\right)\left(\omega+\varepsilon_{1, \xi}-E\right)\left(\omega_{0}+\varepsilon_{2, \xi}-E\right)(2 \omega-E)(\mathrm{A} 6) \\
=g^{2}\left(\varepsilon_{2, \xi}+\varepsilon_{1, \xi}+2 \omega_{0}-2 E\right)^{2} .
\end{gathered}
$$

The spectrum has a more complex structure, see Fig. 1(c) of the main text. The two-excitations subspace provides non-local quantum-dot-cavity states, such as state 16 , which has two trions (one in each dot). The energy of such state is different from the combined energy of two trion states localized in each dot, such as $E_{4}$ and $E_{8}$,

$$
\Delta E_{16,4} \neq \Delta E_{4,0}+\Delta E_{8,0}
$$

where $\Delta E_{n, n^{\prime}}=E_{n}-E_{n^{\prime}}$. This is the basis for the two-qubit conditional phase gate in this work. Using a perturbative approach like that above, we obtain $\Delta E_{16,4}-\left(\Delta E_{4,0}+\Delta E_{8,0}\right) \sim-g^{2} / \Delta \varepsilon$. 


\section{Appendix B: Gate Fidelity}

The fidelity of the gate described in the main text is affected by two type of processes: (i) induced unintended transitions between the states of the qubit-cavity system and (ii) real losses due to cavity leakage and trion recombination. We first estimate losses due to unintended but coherent dynamics. We include transitions 0-4, 4-6, 2-5, 3-7, 0-8, 2-10, 1-9, 3-11 for pulse $A$, and 4-16, 6-18, $0-8,2-10,1-9,3-11$ for pulse B $\left(\omega_{B}\right)$. Other transitions are negligible either due to vanishing matrix elements or to large detuning. We compute the wave function after the A-B-A pulse sequence for each basis configuration of the qubit subspace as initial state (evolution is linear and therefore the resultant wave function for any initial qubit state can be easily recovered). To simplify calculations here we resort to analytically solvable Rosen-Zener pulse shapes, $\frac{131}{1}$ i.e. $\Omega_{p}(t)=\Omega_{p} \operatorname{sech}\left(\sigma_{p} t\right)$ with $\sigma_{A}=2 \sigma_{B}=2 \sigma$, to calculated transition amplitudes and phases for resonant and off-resonance transitions for each pulse. Given the initial, $\left|\psi_{0}\right\rangle$, and final, $|\psi\rangle=U\left|\psi_{0}\right\rangle$, wave function, the fidelity can be computed as

$$
F\left(\psi_{0}, \psi\right)=\left|\left\langle\psi_{0}\left|U_{C Z}^{\dagger}\right| \psi\right\rangle\right|
$$

where $U_{C Z}^{\dagger}$ is the evolution operator corresponding to the ideal $\mathrm{CZ}$ gate. The value of $F\left(\psi_{0}, \psi\right)$ depends on the initial state of the two-qubit system and therefore can vary depending on the choice of algorithm and initial data. We therefore compute the average fidelity $F$ by taking average over all possible initial states of the twoqubit system,

$$
\begin{aligned}
F^{2} & =\int d \psi_{0} F\left(\psi_{0}, \psi\left\{\psi_{0}\right\}\right)^{2} \\
& =\sum_{i j n m} \frac{\delta_{i n} \delta_{j m}+\delta_{i j} \delta_{n m}}{20}\left\langle n\left|U_{C Z}^{\dagger} U\right| i\right\rangle\left\langle j\left|U^{\dagger} U_{C Z}\right| m\right\rangle
\end{aligned}
$$

The integration $\int d \psi_{0}$ is performed over all complex amplitudes that define the initial state in the basis $|i\rangle$, and $i, j, n, m$ run over all basis states $\uparrow \uparrow, \uparrow \downarrow, \downarrow \uparrow, \downarrow \downarrow ! 32$ The results are presented in Fig. 2 and the discussion is given in the main text.

In order to account for both unintended dynamics and actual losses we have to calculate the reduced density matrix, $\rho(t)$, of the two-qubit sub-system for the duration of the pulse sequence. The reduced density matrix can be found within the Bloch-Redfield master-equation (ME) formalism

$$
\begin{aligned}
& i \dot{\rho}=[H+V(t), \rho] \\
& +\sum_{s} i \Gamma_{s}\left[P_{s} \rho P_{s}^{\dagger}-\frac{P_{s}^{\dagger} P_{s} \rho+\rho P_{s}^{\dagger} P_{s}}{2}\right]
\end{aligned}
$$

where $P_{s}=\left|f_{s}\right\rangle\left\langle i_{s}|,| i_{s}\right\rangle$ and $\left|f_{s}\right\rangle$ are initial and finial states (in the spin basis) corresponding to the $s$-th decay process with rate $\Gamma_{s}$. Solving the above equation directly is computationally involving due to the presence of two different time scales: fast, associated with the laser driving frequency, and slow, coming from the timedependence of the pulse shaped envelope. To simplify the computation we transform the ME to the eigenbasis of $H$ and use the rotating wave approximation,

$$
\begin{aligned}
& \dot{\tilde{\rho}}=-i[V(t), \tilde{\rho}] \\
& +\sum_{s} \Gamma_{s}\left[\mathcal{P}_{s} \tilde{\rho} \mathcal{P}_{s}^{\dagger}-\frac{\mathcal{P}_{s}^{\dagger} \mathcal{P}_{s} \tilde{\rho}+\tilde{\rho} \mathcal{P}_{s}^{\dagger} \mathcal{P}_{s}}{2}\right]
\end{aligned}
$$

where $\tilde{\rho}=e^{i \mathcal{H}_{0} t} U^{\dagger} \rho U e^{-i \mathcal{H}_{0} t}, \quad \mathcal{P}_{s}=U^{\dagger} P_{s} U$ and $V(t)=e^{i \mathcal{H}_{0} t} \mathcal{V}(t) e^{-i \mathcal{H}_{0} t}$. Note that the trion decay processes can involve photons with any in-plane polarization (along or perpendicular to the applied magnetic field). Therefore, for each trion we have $\Gamma_{s}=\Gamma_{\mathrm{tr}}$ : $P_{s} \rightarrow\{|\uparrow\rangle\langle\Uparrow|,| \downarrow\rangle\langle\Uparrow|,| \uparrow\rangle\langle\Downarrow|,| \downarrow\rangle\langle\Downarrow|\}$. Leakage of photons from the cavity is modeled as $\Gamma_{s}=\Gamma_{c}: P_{s} \rightarrow$ $\{|0\rangle\langle 1|, \sqrt{2}| 1\rangle\langle 2|$, etc. $\}$. Due to additional (pseudo)spinflip electron-hole recombination processes, more states are involved than for the coherent case discussed above and we include states from 0 to 19 . We chose to use Gaussian pulse shapes $\Omega_{p}(t)=\left(\Omega_{p} / \sqrt{\pi / 2}\right) \exp \left\{-2 t^{2} \sigma_{p}^{2} / \pi^{2}\right\}$ for numerical convenience and apply the same pulse sequence as before with $\sigma_{A}=2 \sigma_{B}=2 \sigma$.

Since a separable quantum wave function is no longer accessible, fidelity has to be defined differently,

$$
F\left(\psi_{0}, \rho\left\{\psi_{0}\right\}\right)=\sqrt{\left\langle\psi_{0}\left|U_{C Z}^{\dagger} \rho U_{C Z}\right| \psi_{0}\right\rangle}
$$

In this case the average fidelity is computed as

$$
F^{2}=\sum \frac{\delta_{i n} \delta_{j m}+\delta_{i j} \delta_{n m}}{20}\left\langle n\left|U_{C Z}^{\dagger} \rho\{|i\rangle\langle j|\} U_{C Z}\right| m\right\rangle
$$

which is the generalization of Eq. $(\mathrm{B} 2)$ for the case of non-unitary evolution of pure initial state. This is possible due to the fact that the evolution of the density matrix is still described by a linear (but non-unitary) superoperator, i.e. $\rho(t)=T \exp \left(-i \int_{0}^{t} d t L_{H}(t)-t L\right)\left|\psi_{0}\right\rangle\left\langle\psi_{0}\right|$, where $L_{H} O=[H, O]$. As a result, the complex coefficients that define initial $\left(\left|\psi_{0}\right\rangle\right)$ and target $\left(U_{C Z}\left|\psi_{0}\right\rangle\right)$ states in the basis $|i\rangle$ can be integrated out in exactly the same way as for Eq. (B2). The results are presented in Figs. 2 and 3 in the main text.

\footnotetext{
* sophia.economou@nrl.navy.mil
}

1 T. D. Ladd, F. Jelezko, R. Laflamme, Y. Nakamura, C. 
Monroe, and J. L. OBrien, Nature (London) 464, 45-53 (2010).

2 J. D. Sterk, L. Luo, T. A. Manning, P. Maunz, C. Monroe, arXiv:1112.4489 (2011).

3 D. Englund, B. Shields, K. Rivoire, F. Hatami, J. Vuckovic, H. Park, and M. D. Lukin, Nano Lett. 10, 39223926 (2010).

4 A. Faraon, P. E. Barclay, C. Santori, K-M. C. Fu, and Raymond G. Beausoleil, Nature Photonics 5, 301 (2011).

5 J. P. Reithmaier, G. Sek, A. Lffler, C. Hofmann, S. Kuhn, S. Reitzenstein, L. V. Keldysh, V. D. Kulakovskii, T. L. Reinecke, and A. Forchel, Nature (London) 432, 197 (2004).

6 D. Press, T. D. Ladd, B. Zhang, and Y. Yamamoto, Nature (London) 456, 218 (2008).

7 A. Greilich, S. E. Economou, S. Spatzek, D. R. Yakovlev, D. Reuter, A. D. Wieck, T. L. Reinecke, and M. Bayer, Nature Physics 5, 262 (2009).

8 T. Yoshie, A. Scherer, J. Hendrickson, G. Khitrova, H. M. Gibbs, G. Rupper, C. Ell, O. B. Shchekin, and D. G. Deppe, Nature (London) 432, 200 (2004).

9 A. Badolato, K. Hennessy, M. Atature, J. Dreiser, E. Hu, P. M. Petroff, and A. Imamoglu, Science 308, 1158 (2005).

10 G. Khitrova, H. M. Gibbs, M. Kira, S. W. Koch, and A. Scherer, Nature Physics 2, 81 (2006).

11 K. Hennessy, A. Badolato, M. Winger, D. Gerace, M. Atatüre, S. Gulde, S. Fält, E. L. Hu, and A. Imamoglu, Nature (London) 445, 896 (2007).

12 D. Pinotsi, P. Fallahi, J. Miguel-Sanchez, and A. Imamoglu, IEEE J. Quant. El. 47, 1371, (2011).

13 S. M. Thon, H. Kim, C. Bonato, J. Gudat, J. Hagemeier, P. M. Petroff, and D. Bouwmeester, arXiv:1109.5016 (2011).

14 E. Gallardo, L. J. Martinez, A. K. Nowak, D. Sarkar, H. P. van der Meulen, J. M. Calleja, C. Tejedor, I. Prieto, D. Granados, A. G. Taboada, J. M. Garcia, and P. A. Postigo, Phys. Rev. B 81, 193301 (2010).

15 S. Reitzenstein, C. Hofmann, A. Gorbunov, M. Strau, S. H. Kwon, C. Schneider, A. Lffler, S. Hfling, M. Kamp, and A. Forchel, Appl. Phys. Lett. 90, 251109 (2007).
16 S. G. Carter, T. M. Sweeney, M. Kim, C. S. Kim, D. Solenov, S. E. Economou, T. L. Reinecke, L. Yang, A. S. Bracker, D. Gammon, arXiv:1211.4540

17 D. Kim, S. G. Carter, A. Greilich, A. S. Bracker and D. Gammon, Nature Physics 7, 223 (2011); A. Greilich, S. G. Carter, D. Kim, A. S. Bracker and D. Gammon, Nature Photonics 5, 702708 (2011).

18 J-Q Zhang, Y-F Yu, and Z-M Zhang, J. Opt. Soc. Am. B 28, 1959 (2011).

19 T. D. Ladd and Y. Yamamoto, Phys. Rev. B 84, 235307 (2011).

20 A. Imamoglu, D. D. Awschalom, G. Burkard, D. P. DiVincenzo, D. Loss, M. Shermin, and A. Small, Phys. Rev. Lett. 83, 4204 (1999).

21 M. A. Nielsen and I. L. Chuang, Quantum Computation and Quantum Information (Cambridge University Press, Cambridge, UK, 2000).

22 M. Notomi, Rep. Prog. Phys. 73 (2010).

23 S. E. Economou, L. J. Sham, Y. Wu, and D. G. Steel, Phys. Rev. B 74, 205415 (2006); S. E. Economou and T. L. Reinecke, Phys. Rev. Lett. 99, 217401 (2007).

24 This assumption is not crucial and can be easily relaxed to accommodate pulses with other polarizations.

25 S. E. Economou and T. L. Reinecke, Phys. Rev. B 78, 115306 (2008).

26 S. Cortez, O. Krebs, S. Laurent, M. Senes, X. Marie, P. Voisin, R. Ferreira, G. Bastard, J-M. Gerard, and T. Amand, Phys. Rev. Lett. 89, 207401 (2002).

27 D. Solenov, D. Tolkunov, and V. Privman, Phys. Rev. B 75, 035134 (2007).

28 E. Waks and C. Monroe, Phys. Rev. A 80, 062330 (2009).

29 J. P. Palao and R. Kosloff, Phys. Rev. Lett. 89, 188301 (2002).

30 S. E. Economou, Phys. Rev. B 85, 241401(R) (2012).

31 N. Rosen and C. Zener, Phys. Rev. 40, 502 (1932).

32 L. H. Pedersena, N. M. Møller, K. Mølmer, Phys. Lett. A 367, 47 (2007). 